\title{
Factors Associated with Emergency Departments in Utilization of Health Services among Minorities who are Enrolled in Medicare
}

\author{
Linda Martinez ${ }^{1 *}$ \\ ${ }^{1}$ Department of Health Care Administration, College of Health and Human Services, California State University, Long \\ Beach, California, USA
}

*Corresponding Author: linda.martinez@csulb.edu

Citation: Martinez, L. (2019). Factors Associated with Emergency Departments in Utilization of Health Services among Minorities who are Enrolled in Medicare. European Journal of Environment and Public Health, 3(2), em0026. https://doi.org/10.29333/ejeph/5852

Published: July 21, 2019

\begin{abstract}
This research was conducted to determine if there is a difference among Medicare and/or Medicaid enrolled patients, based on age, gender and provider diagnosis. The research was conducted using data from the The National Hospital Ambulatory Medical Care Survey (NHAMCS). NHAMCS was designed to collect data on the utilization and provision of ambulatory care services in hospital emergency and outpatient departments and ambulatory surgery locations. Findings are based on a national sample of visits to the emergency departments, outpatient departments, and ambulatory surgery locations of non-institutional general and short hospital stays. The research indicated that there are slightly significant differences between male and female total observations. There are a total of 2471 (53.881\%) females compared to $1855(40.449 \%)$ males for initial visits between the two sexes. For follow-up visits, about $138(3.009 \%)$ are female compared to $122(2.660 \%)$ for males. When looking at the differences between the races, there was a significant difference between white and black. For initial visits, there were 3536 (77.104\%) among whites compared to 646 $(14.086 \%)$ for blacks; others or unknown was $144(3.140 \%)$. Also, there were significant differences for follow-up visits between the two races. About 218 (4.754\%) are white compared to only $36(.0785 \%)$ for black; other race or unknown was only $6(0.131 \%)$.
\end{abstract}

Keywords: medicare, medicaid, ethnicity, race, gender, emergency services

\section{INTRODUCTION}

An emergency room (ER) is a medical treatment facility specializing in emergency medicine and the acute care of patients who arrive at the facility without prior appointment either by their own means or by way of an ambulance. According to the 2013 and 2014 National Health Interview Survey, the use of emergency room (ER) services among U.S. adults stands at $20 \%$. The health insurance types largely associated with ER usage for the adult populations included Medicare, Medicaid and adults who were also uninsured or had private health insurance (Gindi, Black, \& Cohen, 2016). As the U.S. population is becoming more diverse, it is evident that the racial and ethnic composition has implications for the health care systems. There are also many risk factors that affect our health care systems; including, but not limited to health behaviors, disease prevalence, mortality rates, and health insurance coverage. Access to health care and utilization of the health services available to the population differ substantially by race and ethnicity (CDC, 2016).

The Federally Qualified Health Center (FQHC) is a safety net provider in rural areas and provides outpatient clinics that qualify for specific reimbursement systems under Medicare and Medicaid; it is a community-based organization that provides comprehensive primary care and preventive care. The FQHC is a health program 
funded under the Health Center Consolidation Act Section 330 of the Public Health Service Act (1996) and is a reimbursement designation from the Bureau of Primary Health Care (BPHC) and the Centers for Medicare and Medicaid Services (CMS) of the United States Department of Health and Human Services (HHS). There is a relationship between emergency room utilization and access to Federally Qualified Health Centers. It has been determined that emergency room utilization is influenced by several factors. Such factors include, but are not limited to access to primary care within a short distance to FQHCs, a patient's health condition, insurance status, income status, and the closeness of the Emergency Departments versus Federal Qualified Health Centers to the patient's home (CMS, 2018).

Access to the primary care services offered within a Federally Qualified Health Center may result in a negative relationship to the use of the emergency room. The more access an individual may have to routine-care, the less likely they will be to use the emergency room due to the constant monitoring of their health. Conversely, having no access to primary care services in general, whether it be from a publicly funded FQHC or privately funded provider office, will result in a positive relationship to a patient's usage of emergency rooms. When an individual has no access to primary care, they may be more likely to visit the emergency room for treatment; occasionally, the treatment they are seeking could have been sought with their primary care physician. There are 3-types of organizations that are eligible to enroll in Medicaid and/or Medicare as FQHCs according (CMS, 2018). These 3types of organizations are:

- Health Center Program Grantees: Organizations receiving grants under section 330 of the PHS Act, including Community Health Centers, and Public Housing Primary Care Centers;

- Health Center Program Look-Alikes: Organizations that have been identified by HRSA as meeting the definition of "Health Center" under section 330 of the PHS ACT, but not receiving grant funding under section 330; and

- Outpatient health programs/facilities operated by a tribe or tribal organization (under the Indian SelfDetermination Act) or by an urban Indian organization (under Title V of the Indian Health Care Improvement Act, 2017).

According to cms.gov (2018), FQHC services also included certain preventive primary health services and the law defines Medicare-covered preventive services provided by an FQHC as the preventive primary health services that an FQHC is required to provide under section 330 of the Public Health Services (PHS) Act (1996). Research has shown that there is a relationship between access to preventive care, and the need to seek emergency care and due to increased accessibility to primary care services offered within FQHCs, minorities with diabetes will probably visit the emergency room less than those without regular access will to primary care services.

\section{PURPOSE OF THE STUDY}

The purpose of this study is to determine the demographic characteristics among Medicare and/or Medicaid participants utilizing emergency departments. To do this, examining factors such as race, ethnicity, insurance status and previous usage of the emergency department will be analyzed. This study employed secondary data analysis using the 2015 National Hospital Ambulatory Care Survey (NHAMCS) Emergency Department Patient Record dataset. This study also sought to answer the differences among age groups, gender groups, racial groups and insurance status groups in terms of health service utilization. In addition, the study highlighted the varying degrees of medical demands of patients of different races and ethnicities in order to provide more tailored medical services to such patients.

The use of hospital services has been associated generally with ethnicity. The 2008 study conducted by Medicare on Emergency Department (ED) utilization concluded that, $70 \%$ of beneficiaries with six or more chronic conditions had an ED visit and over one-quarter were high ED users (The Medicare Payment Advisory Commission, 2013). Some of the differences identified could be a result of what is considered different health care choices made by the ethnic groups, different distributions of risk factors, as well as disease exposures, and genetic differences that are susceptible to certain diseases. A health care disparity exists when there is unequal access to health information or medical service, causing is a presumably greater clinical need for them.

Early detection and intervention by a Primary Care Physician (PCP) is the key to successful management of several chronic conditions, which ultimately can avoid or minimize the need for emergency admittance and hospitalization. Researchers studying differences in health access and quality of health care have identified numerous conditions that are responsive to ambulatory care. Reasons for hospitalizations for these Ambulatory Care Sensitive (ACS) conditions have indicated poor-quality primary care management is a major issue. There were 15 ACS conditions identified and examined as to whether racial/ethnic groups have more admissions than Caucasian groups. The 15 ACS conditions have been grouped into the following three categories: chronic (includes lung disease, heart failure, diabetes, hypertension), acute (includes bacterial pneumonia, urinary tract infection, 
ulcer, hypoglycemia, ear-nose-throat infections), and preventable conditions (includes malnutrition and influenza) (Mayberry, Berg, Harper, \& Osborn, 2016).

The results show that four percent of Medicare enrollees were admitted to the hospital for one of the above chronic conditions. The Black, American Indian, and Hispanic patients had higher than average hospitalizations for chronic conditions. The Asian/Pacific Islander patients had only a two percent hospitalization rate and about four percent of White patients were hospitalized for chronic conditions. For acute conditions, the average hospitalization rate was also four percent. Black and American Indian patients had higher than average hospitalizations for acute conditions. The Asian/Pacific Islander patients had only two percent hospitalization rate. The White and Hispanic patients' hospitalization rates were at four percent, as well. For preventable conditions, there were only a few differences among the racial/ethnic groups (Mayberry, Berg, Harper, \& Osborn, 2016).

\section{SIGNIFICANCE OF THE STUDY}

There is a need to reduce emergency department utilization through the increase in access to primary care services. There is a correlation between low-income adults and an observed increase in emergency room visits among certain populations of the age group between 46-64 years old with low-income (Agency for Healthcare Research \& Quality, 2017). However, providing enough access to primary care services to the low-income patient has been very challenging, to say the least in the U.S. The Census Bureau in 2016 reported that the top two lowincome racial groups were non-white Hispanics with a mean income of $\$ 47,000$, and African Americans with a mean income of $\$ 39,000$ (U.S. Census Bureau, 2017). Additionally, in 2014, Diabetes Mellitus was amongst the top four chronic conditions present during Emergency Room visits at 9.2\% (CDC, 2014). According Yan, Gushulak, Columbus, Hamelin, van Aarsen, Wells \& Stiell (2017), individuals who utilize emergency departments with a high initial blood glucose level of $>20 \mathrm{mmol} / \mathrm{L}$, were more susceptible to relapse, and may return to the Emergency Room because they represent those diabetic patients who have ongoing poor glycemic control.

According to Shamsihirgaran, Mamaghania, Aliasgarzadeh, Aiminisani, Iranparvar-Alamdari and Ataie (2017), there were total of 387 million people with diabetes in 2013. This figure is predicted to reach 592 million by the year 2035. With an increase in diabetes diagnoses, a strong correlation has been found between the prevalence of diabetes, and various individual demographics, including a person's age, gender, racial ethnicity, BMI, physical activity and dietary patterns. In addition to the aforementioned factors, it was found that the vast majority of these individuals are between the ages of 40 and 59; 80\% of them were identified as living in low and middle income countries (Shamsihirgaran et al., 2017).

\section{RESEARCH METHOD AND INSTRUMENTS}

This study was designed as a secondary data cross sectional study to examine factors associated with Emergency Department (ED) utilization. The National Hospital Ambulatory Medical Care Survey (NHAMCS) collected the data in 2015. The factors included date of visit, race, expected source of payment, diagnosis, and emergency department visits within the last twelve months. These factors were analyzed to determine their relationship with ED utilization. The statistics represent sampling from outpatient, general, and non-federal short stay hospital incidents. The National Ambulatory Medical Care Survey (NAMCS) is designed to meet the need for objective, reliable information about the provision and use of ambulatory medical care services in the United States.

NHAMCS uses a four-stage probability design with samples of primary sampling units (PSUs), hospitals within PSUs, emergency service areas (ESAs) within emergency departments, and patient visits within ESAs. This study examines only visits to the emergency and outpatient departments in the 50 United States and the District of Columbia. NHAMCS looks at both outpatient areas and emergency departments. This study will focus on the NHAMCS Emergency Department element. In the emergency department, the types of service areas used were general, adult, pediatric, fast track and trauma (Centers for Disease Control and Prevention, 2010).

The sampling unit for the 2015 NHAMCS was the patient visit or encounter with the physician. A visit was defined as direct, personal exchange between a patient seeking care and a physician or staff member functioning under the direction of the physician. Non-medical care visits were not included in this study. Clinics are considered eligible for the survey provided their ambulatory medical care is under the supervision of a physician and under the auspices of the hospital. Patient visits within the predefined emergency departments comprised the fourth stage of selection for the study. The hospital staff received training from interviewers to perform data collection for the study. Trained hospital personnel or the Bureau of Census collected the hospital data during the four-week recording period. Visit sampling rates are determined from the expected number of patients to be seen during the reporting period and the desired number of completed Patient Record forms. 


\section{ANALYSIS AND FINDING}

There are three hypothesis to this study and they are the following: (1) There is no difference in ED utilization among Medicare and/or Medicaid enrolled patients based on age, (2) There is no difference of ED utilization among Medicare and/or Medicaid enrolled patients based on gender, and (3) There is no difference of ED utilization among Medicare and/or Medicaid enrolled patients based on provider diagnosis.

Different factors usually influence emergency room usage, including but not limited to health insurance, the level of patient's condition, employment status and income, the proximity of the emergency room compared to Federally Qualified Health Centers distance to the patient's residence. This study utilized the Statistical Packages for Social Sciences version 22 (SPSS) for the analysis. The dependent variable in this study was emergency room utilization while the independent variables were age, gender, health insurance status, race and diagnosis. Fisher's Exact Test was used technically over Chi-Square Test due to some of the cells having an expected value of less than 5 for the age variable. For race and gender, the Chi-Square test was used and those variables are significant.

The total observation data was 4,586 and the researcher analyzed which age categories had a significant difference. Table 1 provides more details based on different age categories. Findings are based on a sample of visits to nonfederal employed office-based physicians, who are primarily engaged in direct patient care and, starting in 2006, a separate sample of visits to community health centers. The National Hospital Ambulatory Medical Care Survey (NHAMCS) is designed to collect data on the utilization and provision of ambulatory care services in hospital emergency and outpatient departments and ambulatory surgery locations. Findings are based on a national sample of visits to the emergency departments, outpatient departments, and ambulatory surgery locations and short-stay hospitals.

The first research question was to determine if there was a difference in emergency department utilization of Medicare patients based on Age. The Pearson Chi-square statistic is 12.125 with a p-value of 0.033 , which is significant at the 0.05 level, but since there are expected counts less than 5 the Fisher's exact test was used instead. Fisher's exact test gives a p-value of 0.045 , which is significant at the 0.05 level. This implies that there is a significant difference in ED utilization of Medicare patients based on age and episode of care.

Table 1. Participants in age categories based on initial and follow-up visit

\begin{tabular}{lccccccc}
\hline Visitation & $\begin{array}{c}\text { Under 15 } \\
\text { years }\end{array}$ & 15- 24 years & $\mathbf{2 4 - 4 4}$ years & $\mathbf{4 5 - 6 4}$ years & $\mathbf{6 5 - 7 4}$ years & $\begin{array}{c}\mathbf{7 5} \text { years and } \\
\text { over }\end{array}$ & Total \\
\hline Initial visit & $31(676 \%)$ & $60(1.308 \%)$ & $408(8.89 \%)$ & $\begin{array}{c}817 \\
(17.815 \%)\end{array}$ & $\begin{array}{c}1193 \\
(26.014 \%)\end{array}$ & $\begin{array}{c}1817 \\
(39.621 \%)\end{array}$ & $\begin{array}{c}4326 \\
(94.331 \%)\end{array}$ \\
\hline $\begin{array}{l}\text { Follow-up } \\
\text { visit }\end{array}$ & $0(0 \%)$ & $8(0.174 \%)$ & $34(0.741 \%)$ & $54(1.177 \%)$ & $62(1.352 \%)$ & $102(2.224 \%)$ & $260(3.669 \%)$ \\
\hline Total & $31(0.676 \%)$ & $68(1.483 \%)$ & $\begin{array}{c}442 \\
(9.638 \%)\end{array}$ & $\begin{array}{c}871 \\
(18.993 \%)\end{array}$ & $\begin{array}{c}127.366 \%) \\
(461.845 \%)\end{array}$ & 4586 \\
\hline
\end{tabular}

The second research question was to determine if there was a difference in utilization of emergency departments based on gender. The Chi-square statistic is 1.634 with a p-value of 0.201 . These values imply that there is not a significant difference in ED utilization of Medicare patients based on gender and episode of care. The final question was to determine if utilization of emergency department was based on race. The Chi-square statistic is 1.11 with a p-value of 0.563. These values imply that there is not a significant difference in ED utilization of Medicare patients based on race (white, black, other) and episode of care.

\section{SUMMARY AND CONCLUSION}

The National Hospital Ambulatory Medical Care Survey (NHAMCS) was designed to collect data on the utilization and provision of ambulatory care services in hospital emergency and outpatient departments and ambulatory surgery locations. Findings are based on a national sample of visits to the emergency departments, outpatient departments, and ambulatory surgery locations of non-institutional general short hospital stays.

The "Episode of care" attempts to measure the nature of the care provided at the visit, an initial visit versus a follow-up visit. An episode of care begins with the initial visit for a particular problem and ends when the patient is no longer continuing treatment. A problem may recur later, but that is considered a new episode of care. An initial visit may be diagnostic in nature, whereas a follow-up visit may be to check progress or continue therapy.

The overall purpose of this study was to determine the Emergency Department (ED) utilization of Medicare enrollees and the method of payment data was used to filter out payers, other than Medicare. Frequency analysis 
was conducted to determine gender, race, age, patient residence, and episode of care in the previous twelve months of care. There are slightly significant differences between male and female total observations. There are a total of $2471(53.881 \%)$ females compared to $1855(40.449 \%)$ males for initial visits between the two sexes. For follow-up visits, about $138(3.009 \%)$ are female compared to $122(2.660 \%)$ for males. When looking at the differences between the races, there was a significant difference between white and black. For initial visits, there were 3536 (77.104\%) among whites compared to $646(14.086 \%)$ for blacks; others or unknown was $144(3.140 \%)$. Also, there were significant differences for follow-up visits between the two races. About 218 (4.754\%) are white compared to only $36(.0785 \%)$ for black; other race or unknown was only six $(0.131 \%)$.

\section{REFERENCES}

Agency for Healthcare Research \& Quality (2017). National healthcare disparities report. AHRQ publication no. 05 0014. Available at: https://www.ahrq.gov/research/findings/nhqrdr/index.html

Center for Disease Control and Prevention (2015). The National Hospital Ambulatory Medical Care Survey (NHAMCS). Available at: https://www.cdc.gov/nchs/ahcd/index.htm

Centers for Disease Control and Prevention (2014). Chronic kidney disease surveillance System—United States. Available at: http://www.cdc.gov/ckd

Centers for Disease Control and Prevention (2016). Health, United States, 2016: With chartbook on long-term trends in bealth. Available at: https://www.cdc.gov/nchs/data/hus/hus16.pdf

Centers for Medicaid and Medicare Services (2018). Medicare benefit policy manual chapter 13 -rural health clinic (RHC) and federally qualified health center (FQHC) services. Table of contents (rev. 239, 01-09-18). Available at: https://www.cms.gov/Regulations-and-Guidance/Guidance/Manuals/Downloads/bp102c13.pdf

Gindi, R. M., Black, L. I., \& Cohen, R. A. (2016). Reasons for emergency room use among U.S. adults aged 18-64: National Health Interview Survey, 2013 and 2014. Available at: https://www.ncbi.nlm.nih.gov/pubmed/26905514

Indian Health Care Improvement Act (2017). Public Law 94-437; Approved September 30, 1976; 25 U.S.C. 1601 et seq.] [As Amended Through P.L. 115-91, Enacted December 12, 2017].

Indian Self-Determination Act (1975). Public Law 93-638.

Mayberry, L. S., Berg, C. A., Harper, K. J., \& Osborn, C. Y. (2016). The design, usability, and feasibility of a familyfocused diabetes self-care support health intervention for diverse, low-income adults with Type 2 Diabetes. Journal of Diabetes Research. https:// doi.org/10.1155/2016/7586385

Medicare Payment Advisory Commission (2016). Report to the Congress: Medicare and the health care delivery system Executive report. Available at: http:/ /www.medpac.gov/docs/default-source/reports/jun13_executivesummary. pde?sfvrsn $=0$

Public Health Service Act, as Amended (42 U.S.C. 247b(k)(2)) (2009).

Shamshirgaran, S. M., Mamaghanian, A., Aliasgarzadeh, A., Aiminisani, N., Iranparvar-Alamdari, M., \& Ataie, J. (2017). Age differences in diabetes-related complications and glycemic control. BMC Endocrine Disorders, 171-7. https://doi.org/10.1186/S12902-017-0175-5

United States Census Bureau (2017). Available at: https://www.census.gov/

Yan, J. W., Gushulak, K. M., Columbus, M. P., Hamelin, van Aarsen, K., Hamelin, A. L. Wells, G. A., \& Stiell, I. G. (2017). Risk factors for recurrent emergency department visits for hyperglycemia in patients with diabetes mellitus. International Journal of Emergency Medicine, 10(1), 23. https://doi.org/10.1186/s12245-017-0150-y 